\title{
ON THE INFECTIVITY OF AN ORIENTAL LUNG FLUKE, PARAGONIMUS OHIRAI MIYAZAKI, 1939 TO SOME GASTROPOD MOLLUSCS, WITH REMARKS ON THAT OF OTHER SPECIES OF THE GENUS IN JAPAN - A REVIEW - \\ KENJIRÔ KAWASHIMA \\ Department of Parasitology, Faculty of Medicine, Kyushu University, Fukuoka, Japan
}

(Received : August 9th, 1965)

\begin{abstract}
Up to the present, the experimental infection with Paragonimus ohirai larvae in the gastropod molluscus belonging to 5 genera including 10 species has been carried out by the author and some other workers. The results showed that this fluke has a high infectivity to amphibious molluscs such as Assiminea parasitologica, A. yoshidayukioi, Paludinella japonica and Oncomelania nosophora rather than to aquatic molluscs. These results were compared with those of the experimental infection with other species of the genus Paragonimus in Japan. The grouping of Paragonimus species of Japan according to their infectivity to the molluscs was coincident with that by the morphological characteristics.
\end{abstract}

\section{INTRODUCTION}

Up to the present, the occurrence of 4 species of the genus Paragonimus has been reported in Japan. P. westermani (KERBERT, 1878) had been recognized for a long time, and another mammalian lung fluke, $P$. ohirai was described as a new species by Miyazaki in 1939. Moreover, P. iloktsuenensis which was described by Chen in 1940 in China was added to the genus Paragonimus of Japan by Miyazaki in 1944. Subsequently, $P$. miyazakii was described by Kamo, Nishida, Hatsushika and Tomimura in 1961 as the fourth species of this genus in Japan. On the other hand, a species of the genus Paragonimus which is closely allied to P. kellicotti WARD, 1908 was reported by Miyazaki in Japan. At present, however, Miyazaki is of the opinion that this species is not referred to as P. kellicotti but as P. miyazakii KAMO et al., 1961.

As regards the molluscan hosts and the intramolluscan development, much information has been accumulated by many earlier workers over the past fifty years. Since Nakagawa first reported in 1915 on the experimental infection of Melania libertina GOULD, 1859 [=Semisulcospira bensoni (PHILIPPI, 1951)] with P. westermani, its infectivity to the molluscs and its intramolluscan stages have been reported by Ando (1915-1921), Nakagawa (1918), Kobayashi (1918-1924), Miyairi (1918, 1934), Yokogawa and Wakeshima (1934), Yamaguti (1943), Komiya and Ito (1950), Komiya, Suzuki and Ito (1961) and Kawashima and Miyazaki (1964b). The molluscan host and the intramolluscan stages of $P$. ohirai were first reported by Ogita (1954). Thereafter, the infectivity of this fluke to the molluscs and its intramolluscan development have been reported by Ikeda (1957), Yokogawa

川島 健治郎 (九州大学医学部寄生虫学教室) 
et al. (1958), Yoshida and Miyamoto (1959, 1960), Yoshida (1960), Kawashima (1961), and Kawashima and Miyazaki (1963a). The larvae of $P$. iloktsuenensis were studied by Chen (1940) in China, and its infectivity to the molluscs and its morphology were also studied by Yoshida (1959), Tomimura, Terauchi and Tarumoto (1960), Komiya, Yoshida and Tomimura (1960) and Kawashima and Miyazaki (1963b). The infectivity of $P$. miyazakii to the molluscs has never been reported except an experimental study by Kawashima and Miyazaki (1964a). Concerning P. kellicotti in north America, Ameel (1934) worked out its life history by experimental infections of American Pomatiopsis molluscs.

In the following pages, the author wishes to review on the infectivity of $P$. ohirai to some gastropod molluscs, together with that of other species of the genus Paragonimus mentioned above.

\section{Experimental Molluscan Infection with P. ohirai Larvae}

Data reported on the experimental infection of some gastropod molluscs with $P$. ohirai larvae are summarized in Table 1.

The experimental infection of Oncomelania nosophora (RoBSON, 1915) was carried out by Kawashima and Miyazaki (1963a) who reported that in all of 66 molluscs examined

Table 1. Comparison of the results of the experimental infections of some gastropod molluses with Paragonimus ohirai MrYAZAKI, 1939

\begin{tabular}{|c|c|c|c|c|c|}
\hline Species & $\begin{array}{l}\text { No. of } \\
\text { molluscs }\end{array}$ & $\begin{array}{l}\text { No. of } \\
\text { infected }\end{array}$ & $\begin{array}{l}\text { Infection } \\
\text { rate } \\
(\%)\end{array}$ & $\begin{array}{l}\text { Days } \\
\text { after } \\
\text { exposure }\end{array}$ & Investigators (year) \\
\hline \multirow[t]{2}{*}{ O. nosophora } & 66 & 66 & 100 & $103-134$ & Kawashima \& Miyazaki (1963) \\
\hline & 22 & 22 & 100 & $1-94$ & Kawashima (Unpublished) \\
\hline A. parasitologica & 30 & 10 & 33.3 & $113-129$ & Yokogawa et al. (1958) \\
\hline \multirow{3}{*}{$\begin{array}{l}=P \text { devilis of } \\
\text { Yokogawa, } 1958\end{array}$} & 69 & 42 & 60.8 & $57-103$ & Yoshida \& Miyamoto (1959) \\
\hline & 14 & 4 & 28.5 & 88 & Kawashima (1961) \\
\hline & 48 & 31 & 64.6 & $85-92$ & Kawashima (Unpublished) \\
\hline \multirow[t]{2}{*}{ A. yoshidayukioi } & 33 & 19 & 57.6 & $87-94$ & Yoshida \& Miyamoto (1960) \\
\hline & 45 & 26 & 57.8 & $85-92$ & Kawashima (Unpublished) \\
\hline P. japonica & 40 & 12 & 30.0 & & Yoshida (1960) \\
\hline \multirow[t]{5}{*}{ A. japonica } & & & + & 63 & Ogita (1954) \\
\hline & & & + & $66-105$ & Ikeda (1957) \\
\hline & 33 & 0 & 0 & $113-129$ & Yokogawa et al. (1958) \\
\hline & 50 & 1 & 2.0 & $57-83$ & Yoshida \& Miyamoto (1959) \\
\hline & 72 & 4 & 5.6 & 100 & Kawashima (Unpublished) \\
\hline A. castanea & 97 & 0 & 0 & $94-138$ & Kawashima (1961) \\
\hline A. kushimotoensis & 37 & 0 & 0 & & Yoshida (1960) \\
\hline \multirow{2}{*}{$\begin{array}{l}\text { A. latericea } \\
\text { miyazakii }\end{array}$} & 44 & 0 & 0 & $88-91$ & Kawashima (1961) \\
\hline & 104 & 0 & 0 & $71-83$ & Kawashima (Unpublished) \\
\hline \multirow[t]{2}{*}{ S. bensoni } & & & - & & Yoshida (1960) \\
\hline & 55 & 0 & 0 & $80-95$ & Kawashima (Unpublished) \\
\hline \multirow[t]{2}{*}{ C. retropuctus } & 10 & 0 & 0 & 102 & Yoshida \& Miyamoto (1959) \\
\hline & 50 & 0 & 0 & $80-100$ & Kawashima (Unpublished) \\
\hline
\end{tabular}


during the period of 103-134 days after exposure to the miracidia, a number of rediae and cercariae of this fluke were found. Moreover, the author observed the various stages of the larvae of this fluke in all 22 molluscs examined during the period of 1-94 days after exposure (unpublished). However, this mollusc may play a very minor role as an intermediate host of $P$. ohirai under the natural condition, because $O$. nosophora and $P$. ohirai are different from each other in the geographical distribution. The experimental infection of Assiminea parasitologica KURODA, 1958* with this fluke was first carried out by Yokogawa and his co-workers who obtained positives in 10 of 30 molluscs examined during the pericd of 113-129 days after exposure. In 1959, Yoshida and Miyamoto experimentally found rediae and cercariae of this fluke in 42 of 69 molluscs, the same species as those reported by Yokogawa et al. (1958), examined during the period of 57103 days after exposure. In 1961 Kawashima also obtained 4 positives in 14 molluscs of $A$. parasitologica examined 88 days after exposure. Thereafter, Kawashima obtained 31 positives in 48 examined during the period of 85-92 days after exposure, using an improved culture box for the maintenance of molluscs (unpublished). Yokogawa et al. (1958), and Yoshida and Miyamoto (1959) proved the natural infection of P. ohirai in the same species of mollusc collected from the area where this fluke occurs. Moreover, it was also reported by Yoshida and Kawashima (1961) that $A$. parasitologica was found abundantly at almost all river mouths in the areas where $P$. ohirai occurs. Considering a series of facts mentioned above, it was shown that $A$. parasitologica was one of the most important molluscan hosts of $P$. ohirai.

Yoshida and Miyamoto (1960) studied the role of A. yoshidayukioi KURODA, 1959 as the molluscan host of $P$. ohirai and obtained 19 positives of 33 molluscs examined during the period of 87-94 days after exposure, and simultaneously found that some molluscs were naturally infected with $P$. ohirai in the area where this fluke occurs. Kawashima also obtained 26 positives in 45 molluscs, the same species as those reported by Yoshida and Miyamoto (1960), examined during the period of 85-92 days after exposure (unpublished). Even though the distribution of this mollusc is not so well known as that of A. parasitologica because it is too small to be easily collected, it is considered important next to A. parasitologica as the molluscan host of this fluke. Though Paludinella japonica (PILSBRY, 1901) was experimentally proved to be infected with P. ohirai (Yoshida, 1960), it seems to play a very minor role as the molluscan host of this fluke.

The fact that A. japonica VON MARTENS, 1877, living in great numbers in the area where $P$. ohirai occurs, can serve as the molluscan hosts of this fluke, was experimentally proved by Ogita in 1954. This fact was experimentally reconfirmed by Ikeda in 1957. No infection rate, however, was shown by either of the workers. In 1958, Yokogawa and his co-workers reported that 33 molluscs of $A$. japonica examined during the period of 113-129 days after exposure were found all negative for the larvae. On the contrary, Yoshida and Miyamoto (1959) found an infected mollusc of 50 A. japonica experimentally exposed and examined during the period of 57-83 days after exposure. In the author's experiment, using the improved culture box for the maintenance of molluscs a number of rediae and cercariae were found in 4 molluscs out of 72 A. japonica examined 100 days after exposure (unpublished). The infection rate was $5.6 \%$ which was 2.8 times as high as that in Yoshida's experiment. Introduction of a new method for culture of

\footnotetext{
* This mollusc was reported as Paludinella devilis (Gould, 1861) by Yokogawa et al. In 1958, Kuroda did not agree with Yokogawa's identification of this mollusc and he considered it to be a new species of the genus Assiminea to which he gave the name A. parasitologica.
} 
molluscs to the experiment may increase not only the survival time but also the infection rate. However, this mollusc has never been found to be naturally infected with this fluke, in spite of the investigation of a great number of the molluscs. This seems to be due to the fact that habitats of $A$. parasitologica and $A$. japonica are so different as to be called "habitat segregation"* and that the larvae of $P$. ohirai have more close relations to A. parasitólogica than A. japonica in the ecological characteristics (Kawashima, Tada and Miyazaki, 1961 a \& b). It seems that the difference in their habitat gives no opportunity for the miracidia of $P$. ohirai to encounter with $A$. japonica under the natural condition. However, if such an opportunity had been provided under the natural condition, some of the larvae might have grown into the cercariae, though the infection rate was very low.

Meanwhile, the host specificity of $P$. ohirai larvae was experimentally investigated, using A. castanea WESTERLUND, 1883 by Kawashima (1961) and using A. kushimotoensis KuRODA, 1958 by Yoshida (1960), but no larvae were recovered in any species of the molluscs. The infectivity of $P$. ohirai to A. latericea miyazakii HABE, 1943 was also observed by the author. But neither rediae nor cercariae were found in 44 molluscs examined during the period of 88-91 days after exposure (Kawashima, 1961). Though the infection experiment using the improved culture box for this mollusc was also carried out by the author, the larvae of this fluke were never recovered in this mollusc (unpublished). The infectivity of P. ohirai to Semisulcospira bensoni (PHILIPPI, 1851) and to Clithon retropictus (VON MARTENS, 1879) was also investigated experimentally, but no larvae were recovered in either species of the molluscs (Yoshida, 1960 ; Yoshida and Miyamoto, 1959 ; Kawashima, unpublished).

\section{Grouping of the Molluscs by Their Susceptibility to P. ohirai}

Considering a series of the results of the experimental infection of some gastropod molluses with $P$. ohirai from the stand point of the host-parasite relationship, these molluscs may be grouped into three. The first group consisting of $A$. parasitologica, $A$. yoshidayukioi, $O$. nosophora and $P$. japonica, the former two species of which were found to be naturally infected with this fluke, is referred to the group of the molluscs which have a high infectivity to this fluke. The second group including A. japonica is referred to the group which has a slight infectivity to the same fluke. The third group consisting of $A$. castanea, A. kushimotoensis, A. latericea miyazakii, S. bensoni and $C$. retropuctus is referred to the group in which the larvae of the fluke were never recovered. From the above-mentioned results, it seems that the difference in the susceptibility to $P$. ohirai, between the first two and the others in the first group may be mainly due to the difference in the habitat between them. If $O$. nosophora or $P$. japonica be found in the area where $P$. ohirai occurs, they would serve as a natural molluscan host of the fluke. The difference in the susceptibility between the first and the second two groups of the molluscs may be also due to the difference in their habitat. It seems that the difference in their habitat results in the reduction of the chance of encounters with $A$. japonica and secondarily in the decrease of the suitability to the mollusc. Then, it is likely that the difference in the susceptibility between the first and the second groups is quantitative. However, as the third group has never been found to be experimentally or naturally infected with this fluke, the differences between the first or the second group

* This phenomenon will be included in "the second type of habitat segregation" that was defined by Miyadi and Mori (1953): Animal ecology, pp. 221, Iwanami-shoten, Tokyo. 
and the third one are qualitative and may be influenced not only by ecological but also by their physiological characteristics.

As mentioned previously, it seems that $P$. ohirai has a higher infectivity to amphibious molluscs such as A. parasitologica, A. yoshidayukioi, $P$. japonica and $O$. nosophora than to aquatic molluses.

\section{Experimental Molluscan Infection with Paragonimus Larvae Except P. ohirai}

It is a very interesting fact that $P$. iloktsuenensis is very similar to $P$. ohirai not only in the crustacean host or the mammalian host but also in the molluscan host. Namely, it was experimentally proved by Yoshida in 1960 that $P$. iloktsuenensis developed into the cercariae in the following 4 species of the Japanese gastropod molluscs: A. parasitologica (infection rate : 56.6\%), A. yoshidayukioi (65.3\%), A. japonica (8.3\%) and $P$. japonica (2.6\%), of which the first species was found to be naturally infected with this fluke by Yoshida in 1959 and by Tomimura, Terauchi and Tarumoto in 1960. In Formosa, the larvae of $P$. iloktsuenensis were found by Chiu (1965) in the mollusc, Tricula chiui HABE et MIYAZAKI, 1962 which is allied to Oncomelania formosana (PILSBRY et HIRASE, 1906). Tang (1940) found a rodent type of Paragonimus, in Fukien, China, and also found the cercariae of the same type in the mollusc, Katayama (=Oncomelania) tangi. In 1963 (b), it was reported by Kawashima and Miyazaki that a number of rediae and cercariae of $P$. iloktsuenensis were found in almost all of $O$. nosophora, in the infection experiment. The above-mentioned molluscs except $A$. japonica have the character to live in the water front and are amphibious. Then, it seems that $P$. iloktsuenensis has a high infectivity to amphibious molluscs as $P$. ohirai. According to Chen (1940), in Canton, China, Assiminea lutea A. ADAMS, 1861 was found naturally infected with $P$. iloktsuenensis. However, there was no information concerning whether or not this mollusc was amphibious.

As to $P$. miyazakii, the author treated it by the same infection method as that used in $P$. ohirai or $P$. iloktsuenensis and also experimentally proved that it developed into the cercaria in $O$. nosophora, though the mollusc showed a slight susceptibility (infection rate : $4.5 \%$; Kawashima and Miyazaki, 1964a). Though there is an opinion that American Pomatiopsis and Japanese Oncomelania should be placed under the same genus, it is interesting that $O$. nosophora collected in Japan was proved by Basch (1959) to be experimentally infected with an American lung fluke, $P$. kellicotti, and that American Pomatiopsis molluscs were proved by Getz (1962) to be also experimentally infected with Schistosoma japonicum (KATSURADA, 1904) from Japan. It is also interesting that hybrids were obtained from experimental crossings of male Pomatiopsis lapidaria and female Oncomelania quadrasi (van der Schalie, Getz and Dazo, 1962).

Semisulcospira bensoni and some other related species have been known as a molluscan host of $P$. westermani which is considered to be one of the most important causative agents of human paragonimiasis. Recently, the infectivity of $P$. westermani to A. parasitologica, A. yoshidayukioi, $P$. japonica, A. kushimotoensis and $O$. nosophora was experimentally investigated. All of them, however, showed negative results for the larvae of this fluke (Yoshida, 1960 ; Kawashima and Miyazaki, 1964 b). The work by Kawashima and Miyazaki (1964b) suggested that it would be doubtful that the cercariae of $P$. westermani were found in O. nosophora in China (Chen, 1941: Nat. Med. Jour. China, 27, 550-552, quoted from Abbott, 1948). 


\section{Grouping of Paragonimus Species by Their Infectivity to the Molluscs}

From the results of studies on the infectivity of Paragonimus to the above-mentioned gastropod molluscs, especially to $O$. nosophora, Paragonimus of Japan, are divided into the following three groups by their infectivity. The first group $-P$. ohirai and $P$. iloktsuenensis - has a high infectivity to amphibious molluscs, especially to O. nosophora, though it has never been found naturally infected. The second group - P. miyazakiihas a slight infectivity to $O$. nosophora but shows no natural infection. The third group - $P$. westermani - has no infectivity to amphibious molluscs including $O$. nosophora but to aquatic molluses such as $S$. bensoni and some other related species. On the other hand, Miyazaki (1964) reported that Paragonimus can be divided into four groups, according to the shape of the ovary and arrangement of cuticular spines of the adult worm, as follow: (1) P. ohiran-P. iloktsuenensis group, ovary profusely branched, cuticular spines in groups; (2) $P$. kellicotti-P. miyazakii group, ovary profusely branched, cuticular spines singly spaced; (3) $P$. westermani group, ovary simply branched off into 6 lobes, cuticular spines singly spaced; (4) P. compactus* group, ovary simply branched off into 6 lobes, cuticular spines in groups. It is a very interesting fact that the grouping of Paragonimus species of Japan by the characteristics of infectivity to the gastropod molluscs was coincident with that by the morphological characteristics mentioned above.

\section{REFERENCES}

AввотT, R. T. (1948): Handbook of medically important mollusks of the Orient and the western Pacific. Bull. of the Mus. of Com. Zool., 100, 245-328.

AMEEL, D. J. (1934): Paragonimus, its life history and distribution in north America and its taxonomy (Trematoda: Troglotrematidae). Am. J. Hyg., 19, 279-317.

BASCH, P. F. (1959): Two new molluscan intermediate hosts for Paragonimus kellicotti. J. Parasitol., 45, 273.

ChEN, H. T. (1940): Morphological and developmental studies of Paragonimus iloktsuensis with some remarks on other species of the genus (Trematoda: Troglotrematidae). Ling. Sci. J., $19,429-530$.

CHIU, J. K. (1965): Tricula chiui Habe et Miyazaki, 1962: A snail host for Paragonimus iloktsuenensis Chen, 1940 in Taiwan. Japan. J. Parasitol., 14, 269-280 (with Japanese summary).

GeTZ, L. L. (1962): Pomatiopsis cincinnatiensis and P. lapidaria as potential intermediate hosts of Schistosoma japonicum. J. Parasitol., 48, 498-499.

HABE, T. (1942): Classification of Japanese Assimineidae. Venus, 12, 32-56 (text in Japanese).

HABE, T. (1943) : Supplmental notes with corrections on the Japanese Assimineidae. Venus, 13, 96-106 (text in Japanese).

HaBe, T. AND MrYAZAKI, I. (1962): Tricula chiui sp. nov., a new snail host of the lung fluke Paragonimus iloktsuenensis Chen in Formosa. Kyushu J. Med. Sci., 13, 47-49.

IKEDA, A. (1957): The experimental study on the larval development of Paragonimus ohiral Miyazaki, 1939 in the first intermediate host, Assiminea japonica. Japan. J. Parasitol., 6, 312-313 (abstract in Japanese).

Kamo, H., Nishida, H., Hatsushika, R. And Tomimura, T. (1961): On the occurrence of a new lung fluke, Paragonimus miyazakii n. sp. in Japan (Trematoda: Troglotrematidae). Yonago Acta Medica, 5, 43-52.

KAWASHIMA, K. (1961) : An experiment on the host specificity of Paragonimus ohirai Miyazaki, 1939 to several snails of the genus Assimines. Japan. J. Papasitol., 10, 161-164 (text in

* P. compactus has not been found in Japan. 
Japanese with English summary).

Kawashima, K., TADA, I. AND MryazAkI, I. (1961 a) : Host preference of miracidia of Paragonimus ohirai Miyazaki, 1939 among three species of snails of the genus Assiminea. Kyushu J. Med. Sci., 12, 99-106.

KAWAShima, K., TADA, I. AND MIYAzAKI, I. (1961 b) : Ecological analysis on the mechanism of the host preference of miracidia of Paragonimus ohirai Miyazaki, 1939 in natural condition. Kyushu J. Med. Sci., 12, 143-151.

KAWASHIMA, K. AND MryazAKI, I. (1963a): The experimental infection of larval lung-fluke to Japanese Oncomelania snails I. Experimentral infection of Paragonimus ohirai Miyazaki, 1939 to Oncomelania nosophora (Robson, 1915). Japan. J. Parasitol., 12, 94-97 (text in Japanese with English summary).

Kawashima, K. AND Mryazaki, I. (1963b): The experimental infection of larval lung-fluke to Japanese Oncomelania snails II. Experimental infection of Paragonimus iloktsuenensis Chen, 1940 to Oncomelania nosophora (Robson, 1915). Japan. J. Parasitol., 12, 159-161 (text in Japanese with English summary).

KAWAshima, K. AND MiYAZAKI, I. (1964a). The experimental infection of larval lung-fluke to Japanese Oncomelania snails. III. Experimental infection of Paragonimus miyazakii Kamo, Nishida, Hatsushika and Tomimura, 1961 to Oncomelania nosophora (Robson, 1915). Japan. J. Parasitol., 13, 421-426 (text in Japanese with English summary).

KAWASHIMA, K. AND MiYAZAKI, I. (1964 b): The experimental infection of larval lung-fluke to Japanese Oncomelania snails. IV. Experimental infection of Paragonimus westermani (Kerbert, 1878) to Oncomelania nosophora (Robson, 1915). Japan. J. Parasitol., 13, 459-463 (text in Japanese with English summary).

KomIYA, Y., SUZUKI, R. AND ITO, Y. (1961): The experimental infection of Paragonimus westermani to the first intermediate host, Semisulcospira libertina. I. Experimental infection through the skin penetration of the snail body. Japan. J. Parasitol., 10, 65-70 (text in Japanese with English summary).

KURODA, T. (1958): On the more species of Assiminea from Japan (A fresh water gastropodous genus). Venus, 20, 16-22.

KuRODA, T. (1959): Another new species of Assiminea, a trematode's intermediate host snail from Japan. Venus, 20, 335-338.

MrYAZAKI, I. (1939): Ein neues Lungendistom Paragonimus ohirai n. sp. Fukuoka Acta Medica, 32, 1247-1252 (text in Japanese with German summary).

MrYAZAKI, I. (1944): On the third species of lung fluke from Japan, "small type of Paragonimus ohirai". Report of Kagoshima Medical School, No. 1. 19-25 (text in Japanese).

MrYAZAKI, I. (1955) : Paragonimus kellicotti Ward, 1908 found in the Japanese weasel, Mustela sibilica in Kyushu. Medicine and Biology, 37, 71-74 (text in Japanese).

MtYAZAKI, I., KAWASHIMA, K. AND YoshIDA, Y. (1960): Studies on the snail hosts of Paragonimus ohirai Miyazaki, 1939 and P. iloktsuenensis Chen, 1940. Kyushu J. Med. Sci., 11, 261-275.

MryazAKI, I. (1964) : Further studies on Paragonimus in Japan. Progress of Medical Parasitology in Japan, 4, 457-467 (text in Japanese).

OGITA, K. (1954): A study on the first intermediate host of the lung-fluke, Paragonimus ohirai Miyazaki, 1939. Acta Medica, 24, 148-159 (text in Japanese with English summary).

TANG, C. C. (1940): A comparative study of two types of Paragonimus occurring in Fukien, South China. Chinese Med. J., Supplement 3, 267-291.

Tomimura, T., Terauchi, J. AND Tarumoto, I. (1960): Studies on the first intermediate host of lung fluke, Paragonimus iloktsuenensis Chen, 1940 in the mouth of the River Shinyodo, Osaka Prefecture. Medicine and Biology, 54, 45-51 (text in Japanese).

vaN DeR Schalie, H., GeTz, Lowell L. AND Dazo, Bonifacio C. (1962): Hybrids between American Pomatiopsis and Oriental Oncomelania snails. Am. J. Trop. Med. Hyg., 11, 418-420. 
Yokogawa, M., Yoshimura, H., Koyama, C., Sano, M., Tsuda, M., Suzuki, J. And TsujI, M. (1958) : On a new intermediate host, Paludinella devilis (Gould, 1861) Habe 1942, of Paragonimus ohirai Miyazaki, 1939. Tokyo Ijishinshi, 75, 67-72 (text in Japanese).

Yoshida, Y. AND MiYamoto, M. (1959): Studies on a first intermediate host, Assiminea parasitologica Kuroda, 1958 ("Paludinella devilis", of Yokogawa and Koyama et al. non Gould), of Paragonimus ohirai Miyazaki, 1939. Japan. J. Papasitol., 8, 122-129 (text in Japanese with English summary).

YosHIDA, Y. (1959): Studies on the first intermediate host of Paragonimus iloktsuenensis Chen, 1940 in Japan (1) Experimental infection with the larva of $P$. iloktsuenensis to Assiminea parasitologica Kuroda, 1958 and Assiminea japonica von Martens, 1877. Japan. J. Parasitol., 8, 822-828 (text in Japanese with English summary).

YoshidA, Y. AND MiYamoto, M. (1960): Another new first intermediate host, Assiminea yoshidayukioi Kuroda, 1959 of Paragonimus ohirai Miyazaki, 1939. Japan. J. Parasitol., 9, 211-216 (text in Japanese with English summary).

YosHidA, Y. (1960): Experimental studies on the first intermediate hosts of Paragonimus westermanii, P. ohirai and P. iloktsuenensis. Japan. J. Parasitol., 9, 377-378 (abstract in Japanese).

Yoshida, Y. ANd Kawashima, K. (1961): On the distribution of the snail hosts of Paragonimus ohirai Miyazaki, 1939 and Paragonimus iloktsuenensis Chen, 1940 in Japan. Japan. J. Parasitol., 10, 152-160 (text in Japanese with English summary). 

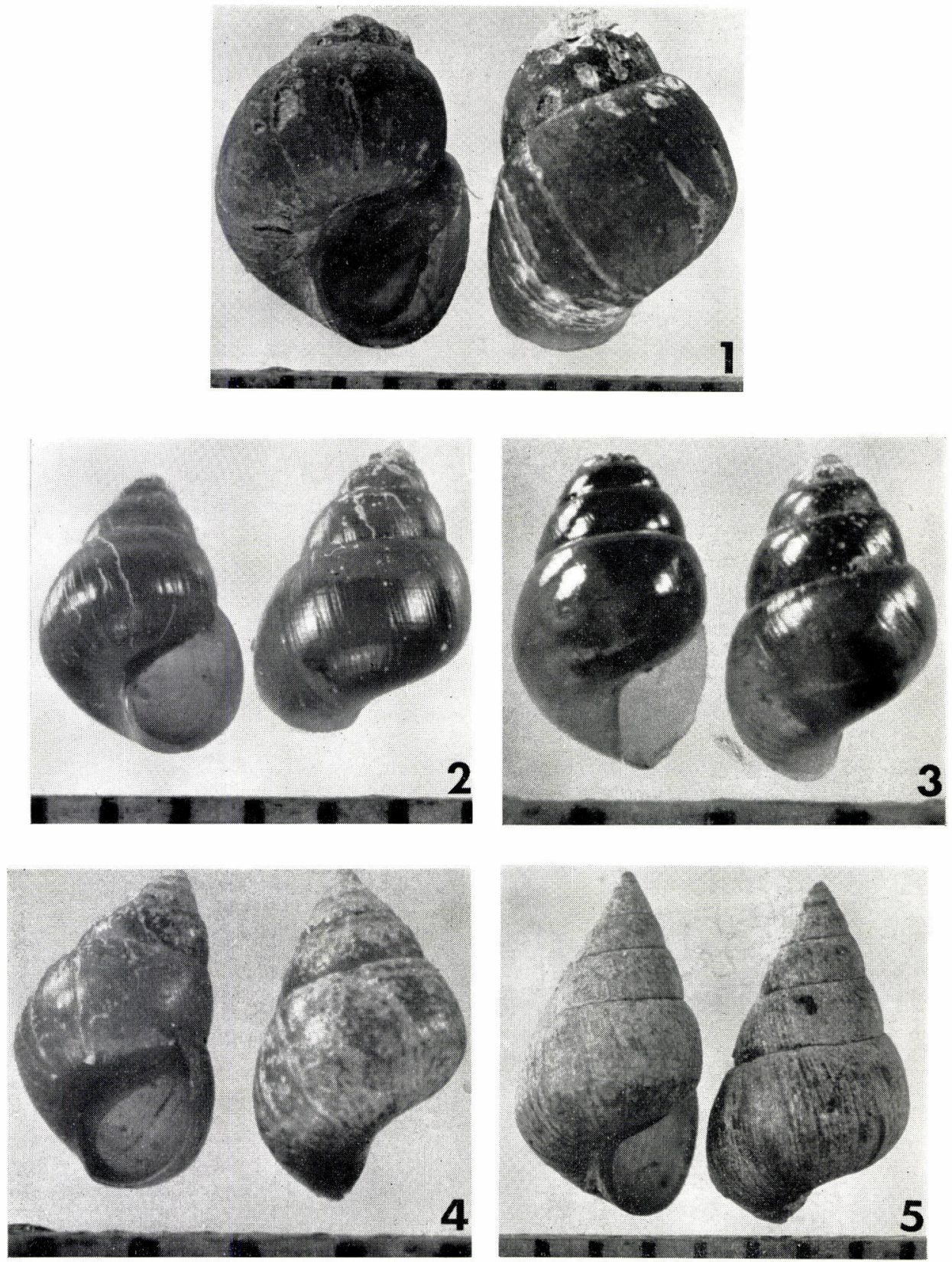

Fig. 1. Assiminea japonica von MARTEns, 1877 (Scale $1 \mathrm{~mm}$

Fig. 2. Assiminea parasitologica KURODA, 1958 (Scale $1 \mathrm{~mm}$ )

Fig. 3. Assiminea yoshidayukioi KURODA, 1959 Scale $1 \mathrm{~mm}$

Fig. 4. Assiminea castanea Westerlund, 1883 (Scale $1 \mathrm{~mm}$ )

Fig. 5. Assiminea kushimotoensis KURODA, 1958 (Scale $1 \mathrm{~mm}$ ) 

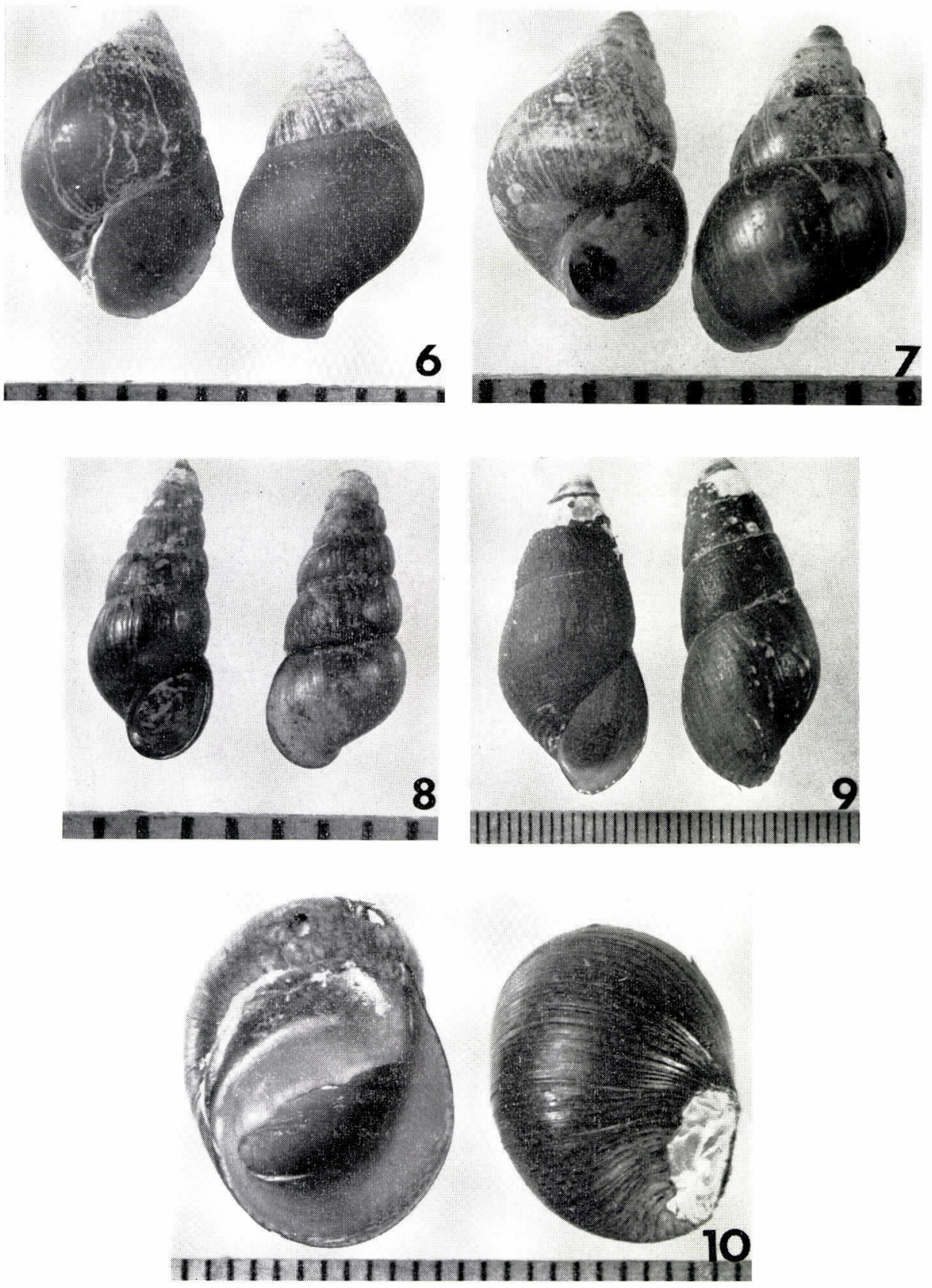

Fig. 6. Assiminea latericea miyazakii HABE, 1943 (Scale $1 \mathrm{~mm}$ )

Fig. 7. Paludinella japonica (PILsBry, 1901) (Scale $1 \mathrm{~mm}$ )

Fig. 8. Oncomelania nosophora (RoBson, 1915) (Scale $1 \mathrm{~mm}$

Fig. 9. Semisulcospira bensoni (PHILIPPI, 1851) (Scale $1 \mathrm{~mm}$ )

Fig. 10. Clithon retropictus (VON MARTENs, 1879) (Scale $1 \mathrm{~mm}$ ) 